\title{
Unbounded solutions of positively damped Liénard equations
}

\author{
by Changming Ding (Hangzhou)
}

\begin{abstract}
This paper discusses the asymptotic behavior of solutions of the Liénard equation, especially the global behavior of unbounded solutions, and also gives a class of sufficient and necessary conditions for the orbit of a solution to intersect the vertical isocline.
\end{abstract}

1. Introduction. In this article we are concerned with the global asymptotic behavior of solutions of the scalar Liénard equation

$$
x^{\prime \prime}+f(x) x^{\prime}+g(x)=0 \quad\left({ }^{\prime}=d / d t\right),
$$

where $f, g: \mathbb{R} \rightarrow \mathbb{R}$ are continuous and satisfy $f(x)>0$ for all $x$ and $x g(x)>0$ for $x \neq 0$. We also assume the regularity for $f(x)$ and $g(x)$ which ensures the existence of a unique solution to the initial value problem.

It is easy to see that the only critical point $(0,0)$ of the equivalent system

$$
x^{\prime}=y, \quad y^{\prime}=-f(x) y-g(x)
$$

is uniformly asymptotically stable, and is globally uniformly asymptotically stable if $\int_{0}^{x} g(s) d s \rightarrow \infty$ as $x \rightarrow \infty$ and $x \rightarrow-\infty$, or $\int_{0}^{x} f(s) d s \rightarrow \infty(-\infty)$ as $x \rightarrow \infty(-\infty)$.

Seifert [1] gives a class of systems (2) for which there exist unbounded solutions which certainly do not approach $(0,0)$ as $t \rightarrow \infty$. If $(x(t), y(t))$ solves (2) with $(x(0), y(0))=(0, a)$, Seifert's main result [1, Theorem 2] says there exist $a_{0}$ and $a_{1}, 0<a_{0} \leq a_{1} \leq \infty$, such that:

(i) $a \geq a_{1}$ implies $y(t)>0$ for $t \geq 0$ and $\lim _{t \rightarrow \infty}(x(t), y(t))=(\infty, L(a))$.

(ii) $a_{0} \leq a<a_{1}$ implies there exist $t_{1}(a)>0$ and $L(a) \leq 0$ such that $y(t)>0$ for $0 \leq t<t_{1}(a), x\left(t_{1}(a)\right)>0, y\left(t_{1}(a)\right)=0, y(t)<0$ for $t>t_{1}(a)$, and $\lim _{t \rightarrow \infty}(x(t), y(t))=(-\infty, L(a))$.

1991 Mathematics Subject Classification: Primary 34C11.

Key words and phrases: unbounded solutions, vertical isocline, global asymptotic behavior. 
(iii) $0 \leq a<a_{0}$ implies $\lim _{t \rightarrow \infty}(x(t), y(t))=(0,0)$.

Concerning the function $L: \mathbb{R}^{+} \rightarrow \mathbb{R}$, Seifert [1] proposed the following questions:

(I) Can $L\left(a_{1}\right)>0$ ? If so, under what conditions will $L\left(a_{1}\right)=0$ ?

(II) Is $L(a)$ strictly increasing for $a \geq a_{1}$ ? Again, if not, are there conditions under which it is?

We note that (1) or (2) has another equivalent system

$$
x^{\prime}=y-F(x), \quad y^{\prime}=-g(x),
$$

where $F(x)=\int_{0}^{x} f(s) d s$. It is also easy to see that the existence of $a_{1}<\infty$ is closely related to the intersection of orbits of (3) and the vertical isocline $y=F(x)$.

In Section 2, we give a simple discussion concerning the relation of systems (2) and (3).

In Section 3 we present a counterexample to a conclusion of Villari [2, Theorem 1], which is also valid for [3, Theorem 2.1]. We give corrections to these theorems and improve the result of [1, Theorem 1].

In Section 4, we answer Seifert's questions completely, i.e., we show that $L\left(a_{1}\right)=0$ and $L(a)$ is strictly increasing for $a \geq a_{1}$.

2. Conjugacy. Put $x=u, y=v-F(u)$ into (2). We have

$$
u^{\prime}=v-F(u), \quad v^{\prime}=-g(u) .
$$

Define $H: \mathbb{R}^{2} \rightarrow \mathbb{R}^{2}$ by $H(x, y)=(x, y+F(x))$. Obviously, $H$ is an isometric homeomorphism and takes orbits of (2) to orbits of (4) (or (3)) preserving their orientation and the parameter $t$, that is, systems (2) and (3) are conjugate. We note that the $y$-axis of the phase plane of system (2) stays invariant under $H$, but the $x$-axis turns to be the vertical isocline $y=F(x)$ of (3), which we denote by $\alpha$.

If $(x(t), y(t))$ solves (3) with $(x(0), y(0))=(0, a)$, let $P=(0, a)$ and denote by $\gamma^{+}$the positive semi-orbit of $(x(t), y(t))$. The basic condition $f(x)>0$ for all $x$ implies $F(x)=\int_{0}^{x} f(s) d s>0$ for $x>0$. The monotonicity of solutions of (3) in the variant regions of the phase plane easily leads to the conclusion that $F(\infty)=\int_{0}^{\infty} f(s) d s<\infty$ is a necessary condition for $\gamma^{+}(P)(P=(0, a), a>0)$ not to intersect the vertical isocline $\alpha$.

Proposition 1. If $f(x)>0$ for all $x$, then $a_{1}<\infty$ implies

$$
\int_{0}^{\infty} f(s) d s<\infty \text { and } \int_{0}^{\infty} g(s) d s<\infty .
$$

Proof. We only need to prove $\int_{0}^{\infty} g(s) d s<\infty$. Otherwise, it is easy to 
see that the curves defined by

$$
V(x, y)=\frac{1}{2} y^{2}+G(x)=\text { constant }
$$

are closed, and since $V^{\prime}=-f(x(t)) y^{2}(t)$ along the solution $(x(t), y(t))$ of system (2), the orbits of (2) are bounded by these closed curves and guided to the positive $x$-axis.

Now by means of $H$ one may thus restate Seifert's questions as follows:

(I) Can $L_{1}\left(a_{1}\right)>F(\infty)(<\infty)$ ? $\left(L_{1}(a)=L(a)+F(\infty)\right)$.

(II) Is $L_{1}(a)$ strictly increasing for $a \geq a_{1}$ ?

3. An example. For system (3) Villari [2, Theorem 1] proves:

Theorem A. Let $F(x)>-c>-\infty$ for $x>0$. For every $\left(x_{0}, y\right)$ with $x_{0} \geq 0$ and $y>F\left(x_{0}\right)$, the orbit of $(3)$ which passes through $\left(x_{0}, y\right)$ intersects the curve $y=F(x)$ at $(x, F(x))$ with $x>x_{0}$ if and only if

$$
\limsup _{x \rightarrow \infty}[G(x)+F(x)]=\infty .
$$

As a counterexample to the theorem we consider a concrete Liénard system

$$
x^{\prime}=y-\left(1-e^{-x}\right), \quad y^{\prime}=e^{-2 x},
$$

so $F(x)=1-e^{-x}$ and $G(x)=\int_{0}^{x} g(s) d s=\left(1-e^{-2 x}\right) / 2$. Then

$$
\lim _{x \rightarrow \infty}[G(x)+F(x)]=\lim _{x \rightarrow \infty}\left[1-e^{-x}+\left(1-e^{-2 x}\right) / 2\right]=3 / 2<\infty .
$$

But for $P=(0, a)$ with $0<a<1, \gamma^{+}(P)$ must intersect the curve $y=F(x)$.

Let

$$
\begin{aligned}
K & =\sup (F(x): x \geq 0), & P & =\lim _{x \rightarrow \infty} G(x), \\
K^{\prime} & =\inf (F(x): x<0), & P^{\prime} & =\lim _{x \rightarrow-\infty} G(x),
\end{aligned}
$$

where $K, P$ and $P^{\prime}$ may be $\infty$ and $K^{\prime}$ may be $-\infty$. We derive the following result as a remedy for Theorem A.

Theorem 1. Suppose $F(x)>-c>-\infty$ for $x>0$ and $F(x)<c<\infty$ for $x<0$. For every $\left(x_{0}, y\right)$ with $x_{0} \geq 0$ and $y>K+(2 P)^{1 / 2}$ the orbit of system (3) passing through $\left(x_{0}, y\right)$ intersects the curve $y=F(x)$ at $(x, F(x))$ with $x>x_{0}$ if and only if $\lim \sup _{x \rightarrow \infty}[G(x)+F(x)]=\infty$.

For every $\left(x_{0}, y\right)$ with $x_{0}<0$ and $y<K^{\prime}-\left(2 P^{\prime}\right)^{1 / 2}$ the orbit of system (3) passing through $\left(x_{0}, y\right)$ intersects the curve $y=F(x)$ at $(x, F(x))$ with $x<x_{0}$ if and only if $\lim \sup _{x \rightarrow-\infty}[G(x)-F(x)]=\infty$.

Proof. Let $\alpha$ be the curve $y=F(x)$. We only consider the case $y>$ $K+(2 P)^{1 / 2}$ with $x_{0} \geq 0$.

Assume that $\lim \sup _{x \rightarrow \infty}[G(x)+F(x)]<\infty$. This implies that $-c<$ $F(x) \leq K<\infty$ and $0<G(x)<P<\infty$ for $x>x_{0}$. 
Consider the curves defined by

$$
V(x, y)=\frac{1}{2}(y-K)^{2}+G(x)=\text { constant } .
$$

It is easy to see that if $G(x)$ has no upper bound these curves are closed, but if $G(x)<P$ the curves which intersect the $y$-axis with $y>K+(2 P)^{1 / 2}$ do not intersect the line $y=K$.

The time rate of change of $V$ along a solution orbit is given by

$$
V^{\prime}=g(x)[K-F(x)] .
$$

Since $F(x) \leq K$, in $x>x_{0}$ the orbits of system (3) do not cross these curves from their exteriors to their interiors. Thus, if $y>K+(2 P)^{1 / 2}$ the orbit of system (3) which passes through $\left(x_{0}, y\right)$ is bounded away from $\alpha$.

Now assume that $\lim \sup _{x \rightarrow \infty}[G(x)+F(x)]=\infty$.

If $\limsup _{x \rightarrow \infty} F(x)=\infty$, the orbit of system (3) which passes through $\left(x_{0}, y\right)$ with $y_{0}>F\left(x_{0}\right)$ obviously intersects $\alpha$.

If limsup $\sup _{x \rightarrow \infty} G(x)=\infty$, consider the closed nested ovals

$$
W(x, y)=\frac{1}{2}(y+c)^{2}+G(x)=\text { constant } .
$$

Since $W^{\prime}=-g(x)[F(x)+c]<0$ if $x>x_{0}$, the orbit passing through $\left(x_{0}, y\right)$ is bounded by the same ovals and guided to $\alpha$.

In exactly the same way we can treat the case $y<K^{\prime}-\left(2 P^{\prime}\right)^{1 / 2}$ with $x_{0}<0$.

Remark 1 . The condition $f(x)>0$ for all $x$ is unnecessary in Theorem 1.

R e mark 2. The result of [3, Theorem 2.1] requires a modification as in our Theorem 1.

By Theorem 1, we easily conclude:

Theorem 2. Suppose $f(x)>0$ for all $x$. Then there exist unbounded solutions of system (3) if and only if

$$
\lim _{x \rightarrow \infty}[G(x)+F(x)]<\infty \quad \text { or } \quad \lim _{x \rightarrow-\infty}[G(x)-F(x)]<\infty .
$$

P r o of. Sufficiency follows from Theorem 1. We only need to prove the necessity. Assume $\lim _{x \rightarrow \infty}[G(x)+F(x)]<\infty$ and $\lim _{x \rightarrow-\infty}[G(x)+F(x)]$ $<\infty$. We consider the positive semi-orbit $\gamma^{+}$of system (3) which passes through $\left(x_{0}, y_{0}\right)$ with $y_{0}>F\left(x_{0}\right)$ and $x_{0} \geq 0$. If $\lim _{x \rightarrow \infty} F(x)=\infty$, the monotonicity of solutions in the phase plane implies $\gamma^{+}$intersects the curve $\alpha$. If $\lim _{x \rightarrow \infty} G(x)=\infty$, consider the closed nested ovals

$$
V(x, y)=\frac{1}{2} y^{2}+G(x)=\text { constant } .
$$

Since $V^{\prime}=-g(x) F(x)<0$ for $x>x_{0}, \gamma^{+}$is bounded by these ovals and guided to $\alpha$. For $\left(x_{0}, y_{0}\right)$ with $x_{0}<0$ and $y_{0}>F\left(x_{0}\right)$, from $d y / d x=$ $-g(x) /(y-F(x))$, it is easy to see that $\gamma^{+}$intersects the positive $y$-axis. 
In exactly the same way we can treat the case $y_{0}<F\left(x_{0}\right)$. Thus, we conclude that for every $P=\left(x_{0}, y_{0}\right) \in \mathbb{R}^{2}, \gamma^{+}(P)$ encircles the origin $(0,0)$. Moreover, $d V(x(t), y(t)) / d t=-g(x(t)) F(x(t))$ implies that $\gamma^{+}$tends to $(0,0)$ as $t \rightarrow \infty$, that is, all solutions of (3) are bounded. This completes the proof.

Remark 3. Theorem 2 improves [1, Theorem 1].

4. The functions $L_{1}(a)$ and $L(a)$. To answer Seifert's first question, we use its restatement in Section 2.

Theorem 3. $L_{1}\left(a_{1}\right)=F(\infty)=\int_{0}^{\infty} f(s) d s(<\infty)$.

Proof. By Proposition 1 of Section 2 let $K=F(\infty)$ and $H=G(\infty)$, and suppose $L_{1}\left(a_{1}\right)>F(\infty)$. We fix $\varepsilon=\left(L_{1}\left(a_{1}\right)-K\right) / 2>0$. Denote by $\beta$ the upper component $y=\varphi(x)$ of $(y-K)^{2} / 2+G(x)=H$. It easily follows that $\lim _{x \rightarrow \infty} \varphi(x)=K$, which implies that there exists a sufficiently large $x_{0}$ satisfying $\varepsilon^{2} / 2+G\left(x_{0}\right)=H$. Let $P=\left(x_{0}, \varepsilon+K\right)$. Because the orbits of (3) cross $\beta$ upwards, the negative semi-orbit $\gamma^{-}(P)$ passing through $P$ will intersect the $y$-axis at $Q=(0, k)(k>0)$, and $\gamma^{+}(P)$ does not intersect the curve $y=K$. Thus, we easily obtain $k>a_{1}$. On the other hand, the monotonicity of $\gamma^{+}(P)$ implies $L_{1}(k)<K+\varepsilon<L_{1}\left(a_{1}\right)$, which contradicts the definition of $a_{1}$.

To answer Seifert's second question we directly use the system (2).

TheOREM 4. $L(a)$ is strictly increasing for $a \geq a_{1}$.

Proof. Let $e>k \geq a_{1}$, and denote by $y=y_{1}(x), y=y_{2}(x)$ respectively the solutions of system $(2)$ which pass through $(0, e)$ and $(0, k)$, that is,

$$
\frac{d y_{1}(x)}{d x}=-f(x)-\frac{g(x)}{y_{1}(x)}, \quad \frac{d y_{2}(x)}{d x}=-f(x)-\frac{g(x)}{y_{2}(x)} .
$$

Therefore

$$
\frac{d\left(y_{1}(x)-y_{2}(x)\right)}{d x}=\frac{g(x)}{y_{1}(x) y_{2}(x)}\left(y_{1}(x)-y_{2}(x)\right) .
$$

Hence $y_{1}(x)-y_{2}(x)$ is increasing as $x$ increases, which leads to

$$
L(e)-L(k)>e-k>0 .
$$

This completes the proof.

Acknowledgements. The author is grateful to the referee for some useful remarks and corrections. 


\section{References}

[1] G. Seifert, Global asymptotic behavior of solutions of positively damped Liénard equations, Ann. Polon. Math. 51 (1990), 283-288.

[2] G. Villari, On the qualitative behavior of solutions of Liénard equations, J. Differential Equations 67 (1987), 269-277.

[3] G. Villari and F. Zanolin, On a dynamical system in the Liénard plane. Necessary and sufficient conditions for the intersection with the vertical isocline and applications, Funkc. Ekvac. 33 (1990), 19-38.

Department of Mathematics

Hangzhou Teachers' College

Hangzhou, 310012, P.R. China

Reçu par la Rédaction le 15.11.1994

Révisé le 20.8.1995 